\title{
Histidine Supplementation for Treatment of Anaemia of Uraemia
}

\author{
M. J. BLUMENKRANTZ, D. J. SHAPIRO, \\ M. E. SWENDSEID, J. D. KOPPLE
}

Characteristics of the 16 chronically uraemic patients and 26 patients on dialysis who completed the study are shown in tables I and II. Patients undergoing haemodialysis received treatment five or six times every two weeks. They underwent dialysis with a single pass fluid Summary

The effects of supplemental histidine on the anaemia of 16 chronically uraemic patients and 26 patients undergoing maintenance dialysis were assessed. Patients were given either L-histidine $4 \mathrm{~g}$ /day or placebo for a mean of 17.5 weeks in a randomized double-blind controlled study. Haemoglobin and packed cell volume increased slightly and to a similar degree in the patients on dialysis receiving placebo and histidine, but this change was statistically significant only in those on placebo. Plasma histidine rose significantly in the histidine-treated patients undergoing dialysis and decreased in the uraemic patients receiving placebo. These results suggest that supplementary $\mathrm{L}$-histidine in patients with uraemia or patients undergoing maintenance dialysis does not improve anaemia.

\section{Introduction}

Histidine is an essential amino-acid for both chronically uraemic patients and healthy people. ${ }^{1-3}$ Diets deficient in histidine are associated with development of anaemia in normal people and progression of anaemia in patients with chronic renal failure. When histidine is added to such diets haemoglobin production rises and the anaemia improves. ${ }^{2} 3$ Plasma histidine levels are decreased in uraemic patients, and possibly histidine deficiency may contribute to the anaemia of uraemia. ${ }^{4}$ Giordano et al. have reported that haematocrit values increased in chronically uraemic patients given oral supplements of dietary histidine. ${ }^{56}$ Anaemia is a serious complication of uraemia and contributes to the morbidity and debility of patients with renal failure. We therefore conducted a double-blind controlled study to evaluate the effects of supplementary histidine on the anaemia of patients with uraemia.

\section{Patients}

Only patients who were clinically stable and had not been in hospital, undergone surgical procedures, or lost significant amounts of blood during the preceeding two months were accepted into the study. Selection was limited to patients who were willing to adhere to the experimental protocol and had previously complied with medical therapy. Thus 23 chronically uraemic patients and 43 patients undergoing maintenance dialysis entered the study. Twenty-four patients failed to complete the study because of superimposed illness, kidney transplantation, noncompliance, or death, and their data were not analysed. All patients gave their informed consent.

\footnotetext{
Medicine and Research Services, Veterans Administration, Wadsworth Hospital Center and Department of Medicine and School of Public Health, University of California at Los Angeles, California 90073, U.S.A.

M. J. BLUMENKRANTZ, M.D., Assistant Professor of Medicine D. J. SHAPIRO, M.B., M.R.C.P., Fellow in Nephrology

M. E. SWENDSEID, PH.D., Professor of Nutrition and Biological Chemistry J. D. KOPPLE, M.D., F.A.C.P., Assistant Professor of Medicine and Public Health
} delivery system and an Ex-01, Ex-03 (Extracorporeal) or Gambro Lundia dialyser. Two patients (cases 21 and 40) were treated with chronic peritoneal dialysis. ${ }^{7}$ All patients except one on dialysis treatment (case 36) were men. One patient (case 35) was anephric. The placebo and histidine groups were similar in age, weight, cause of renal failure, level of renal function, and duration of azotaemia or dialysis therapy. Eight dialysis patients in each group received a mean of $400 \mathrm{mg} /$ week of testosterone enanthate (Delatestryl) for at least four months before the baseline period and continued to receive it during the study.

All chronically uraemic patients had been azotaemic (creatinine clearance $<30 \mathrm{ml} / \mathrm{min}$ ) for more than one year and 12 patients had been azotaemic for more than two years. During the treatment period serum urea nitrogen values were greater in the histidine group than in the placebo group $(P<0.01)$.

\section{Methods}

The patients were observed for six to eight weeks to ensure that they were clinically stable and to obtain baseline data. They were then assigned, with the use of a table of random numbers, to placebo or treatment groups which received either methylcellulose $2 \mathrm{~g} /$ day or L-histidine as the free base $4 \mathrm{~g}$ /day in identical looking capsules. Both the placebo and histidine were taken in two capsules four times daily-with meals and at bedtime. Eight uraemic patients received histidine and eight received placebo, while 12 dialysis patients were given histidine and 14 placebo.

Six months after the start of the study, baseline data were assessed by an independent observer who did not know the patient. Subsequent entrants into the study were assigned by this observer to either the histidine or placebo group so as to make the initial clinical characteristics of the two groups similar. Neither the patients nor their doctors knew which treatment they were receiving or the results of the study. At the end of treatment selection of data for analysis was performed by a doctor who did not know which group the patient was in. The data were then statistically analysed by independent observers who had no contact with the patients. Data collected during periods of intercurrent illness or blood loss and subsequent convalescence were not analysed.

Compliance with the experimental regimen was assessed by careful questioning, observation of patients, and evaluation of the frequency with which subjects requested repeat prescriptions. Patients who took fewer than four capsules per day were excluded from analysis.

Diet.-The chronically uraemic patients were prescribed at least $40 \mathrm{~g} /$ day of primarily high quality protein; the patients undergoing dialysis were generally prescribed $1 \mathrm{~g} \mathrm{~kg}^{-1}$ day $^{-1}$ of primarily high biological value protein; and diets provided at least $0.15 \mathrm{MJ} \mathrm{kg}$ day $^{-1}\left(35 \mathrm{kcal} \mathrm{kg}^{-1}\right.$ day $\left.^{-1}\right)$. Dietary intake of protein and histidine during the study were estimated by dietary interview techniques with the use of food composition tables. ${ }^{8} 9$ Patients received daily supplements of multivitamins including folic acid $1 \mathrm{mg} /$ day. Most chronically uraemic patients took a daily supplement of iron during the study or were given iron dextran (Imferon) intramuscularly at the start of the baseline assessment. Subjects und rgoing dialysis received iron dextran intravenously when serum iron levels dropped below $600 \mu \mathrm{g} / 1$.

Investigations. - During baseline and treatment periods blood was drawn every two weeks for analysis of haemoglobin, packed cell volume (P.C.V.), white cells, and platelets (Coulter counter). Serum urea nitrogen, creatinine, and iron were assessed once a month with an AutoAnalyser. Plasma histidine was determined in fasting patients twice during the baseline period and during the eighth and 12th week of the treatment period. Histidine was measured with a Beckman Model $121 \mathrm{Hp}$ Amino Acid Analyzer. Vitamin $\mathrm{B}_{12}$ levels were determined with the Clinical Assays kit during the baseline period in 
TABLE I-Clinical Characteristics of Chronically Uraemic Patients Treated With Placebo or L-Histidine. Results are Mean Baseline Values and Values after Six Weeks of Treatment

\begin{tabular}{|c|c|c|c|c|c|c|c|c|}
\hline \multirow{2}{*}{$\begin{array}{l}\text { Case } \\
\text { No. }\end{array}$} & \multirow{2}{*}{$\begin{array}{c}\text { Age } \\
\text { (Years) }\end{array}$} & \multirow{2}{*}{ Renal Disease } & \multirow{2}{*}{$\underset{(\mathrm{kg})}{\text { Weight }}$} & \multicolumn{2}{|c|}{ Creatinine Clearance $(\mathrm{ml} / \mathrm{min})$} & \multicolumn{3}{|c|}{ Serum Urea Nitrogen (mmol/l) } \\
\hline & & & & Baseline & Treatment & Baseline & & Treatment \\
\hline $\begin{array}{l}1 \\
2 \\
3 \\
4 \\
5 \\
6 \\
7 \\
8\end{array}$ & $\begin{array}{l}57 \\
45 \\
56 \\
63 \\
61 \\
60 \\
52 \\
51\end{array}$ & $\begin{array}{l}\text { Interstitial nephritis } \\
\text { Nephrosclerosis } \\
\text { Polycystic kidneys } \\
\text { Nephrosclerosis } \\
\text { Glomerulonephritis } \\
\text { Glomerulonephritis } \\
\text { Glomerulonephritis } \\
\text { Glomerulonephritis }\end{array}$ & $\begin{array}{l}64 \\
82 \\
73 \\
51 \\
75 \\
81 \\
66 \\
68\end{array}$ & $\begin{array}{c}\text { Placebo Group } \\
12 \\
18 \\
5 \\
15 \\
21 \\
23 \\
9 \\
10\end{array}$ & $\begin{array}{r}7 \\
14 \\
4 \\
15 \\
14 \\
22 \\
8 \\
8\end{array}$ & $\begin{array}{l}17 \cdot 9 \\
47 \cdot 1 \\
47 \cdot 8 \\
60 \cdot 7 \\
50 \cdot 7 \\
38 \cdot 6 \\
46 \cdot 4 \\
97 \cdot 8\end{array}$ & & $\begin{array}{l}22 \cdot 9 \\
51 \cdot 4 \\
54 \cdot 3 \\
60 \cdot 7 \\
63 \cdot 6 \\
37 \cdot 1 \\
48 \cdot 6 \\
77 \cdot 8\end{array}$ \\
\hline $\begin{array}{c}\text { Mean } \\
\text { S.D. } \\
\mathbf{P} \text { value } . .\end{array}$ & $54 \pm 61$ & & $70 \pm 10$ & $14 \cdot 1 \pm 6 \cdot 2$ & $<0.0211 .6 \pm 5.7$ & $50 \cdot 7 \pm 22 \cdot 9$ & N.S. & $52 \cdot 1 \pm 17 \cdot 1$ \\
\hline $\begin{array}{r}9 \\
10 \\
11 \\
12 \\
13 \\
14 \\
15 \\
16\end{array}$ & $\begin{array}{l}57 \\
63 \\
50 \\
62 \\
38 \\
64 \\
63 \\
77\end{array}$ & $\begin{array}{l}\text { Nephrosclerosis } \\
\text { Glomerulonephritis } \\
\text { Glomerulonephritis } \\
\text { Lupus nephritis } \\
\text { Glomerulonephritis } \\
\text { Diabetic nephropathy } \\
\text { Lead nephropathy } \\
\text { Pyelonephritis }\end{array}$ & $\begin{array}{l}86 \\
86 \\
84 \\
93 \\
79 \\
68 \\
82 \\
64\end{array}$ & $\begin{array}{c}\text { Histidine Grou } \\
18 \\
23 \\
7 \\
22 \\
16 \\
23 \\
16 \\
10\end{array}$ & $\begin{array}{r}12 \\
17 \\
6 \\
20 \\
10 \\
19 \\
9 \\
13\end{array}$ & $\begin{array}{l}53 \cdot 6 \\
32 \cdot 1 \\
60 \cdot 0 \\
41 \cdot 4 \\
67 \cdot 8 \\
42 \cdot 8 \\
52 \cdot 1 \\
62 \cdot 1\end{array}$ & & $\begin{array}{l}65 \cdot 7 \\
45 \cdot 0 \\
77 \cdot 1 \\
55 \cdot 7 \\
83 \cdot 6 \\
55 \cdot 0 \\
70 \cdot 0 \\
65 \cdot 0\end{array}$ \\
\hline $\begin{array}{c}\text { Mean } \\
\pm \text { S.D. } \\
\text { P value .. }\end{array}$ & $59 \pm 11$ & & $80 \pm 10$ & $16 \cdot 8 \pm 6 \cdot 1$ & $<0.03 \quad 13 \cdot 2 \pm 5.1$ & $51 \cdot 4 \pm 12 \cdot 1$ & $<0.001$ & $64 \cdot 3 \pm 12 \cdot 1$ \\
\hline
\end{tabular}

Conversion: SI to Traditional Units-Serum urea nitrogen: $1 \mathrm{mmol} / 1 \approx 1 \cdot 4 \mathrm{mg} / 100 \mathrm{ml}$.

TABlE II-Clinical Characteristics of Patients On Dialysis Treated With Placebo or L-Histidine. Results are Mean Data Obtained Two and a Half or Three and a Half Days After Dialysis

\begin{tabular}{|c|c|c|c|c|c|c|c|}
\hline $\begin{array}{l}\text { Case } \\
\text { No. }\end{array}$ & $\begin{array}{c}\text { Age } \\
\text { (Years) }\end{array}$ & Renal Disease & $\underset{(\mathbf{k g})^{*}}{\text { Weight }}$ & $\underset{(\mathrm{mmol} / \mathrm{l})}{\text { Serum Urea Nitrogen }}$ & $\underset{(\mu \mathrm{mol} / \mathrm{l})}{\operatorname{Serumine}}$ & $\begin{array}{c}\text { Received Testosterone } \\
\text { Enanthate }\end{array}$ & $\begin{array}{c}\text { Duration of Dialysis } \\
\text { (Months) }\end{array}$ \\
\hline $\begin{array}{l}17 \\
18 \\
19 \\
20 \\
21 \\
22 \\
23 \\
24 \\
25 \\
26 \\
27 \\
28 \\
29 \\
30\end{array}$ & $\begin{array}{l}45 \\
57 \\
43 \\
52 \\
52 \\
33 \\
51 \\
61 \\
24 \\
59 \\
36 \\
42 \\
53 \\
51\end{array}$ & $\begin{array}{l}\text { Nephrosclerosis } \\
\text { Polycystic kidneys } \\
\text { Glomerulonephritis } \\
\text { Unknown } \\
\text { Nephrosclerosis } \\
\text { Glomerulonephritis } \\
\text { Nephrosclerosis } \\
\text { Nephrosclerosis } \\
\text { Glomerulonephritis } \\
\text { Glomerulonephritis } \\
\text { Glomerulonephritis } \\
\text { Nephrosclerosis } \\
\text { Nephrosclerosis } \\
\text { Unknown }\end{array}$ & $\begin{array}{r}78 \\
78 \\
66 \\
73 \\
106 \\
70 \\
81 \\
68 \\
66 \\
73 \\
63 \\
79 \\
74 \\
72\end{array}$ & $\begin{array}{c}\text { Placebo Group } \\
73.5 \\
73.5 \\
59 \cdot 3 \\
90.7 \\
67.9 \\
67 \cdot 1 \\
69 \cdot 3 \\
52.1 \\
70.7 \\
18.6 \\
74.3 \\
67.1 \\
64.3 \\
75.0\end{array}$ & $\begin{array}{l}1476 \\
1644 \\
769 \\
2033 \\
1680 \\
1688 \\
1140 \\
1017 \\
1768 \\
1662 \\
1467 \\
1574 \\
1423 \\
1344\end{array}$ & $\begin{array}{l}\text { Yes } \\
\text { Yes } \\
\text { No } \\
\text { No } \\
\text { Yes } \\
\text { Yes } \\
\text { Yes } \\
\text { No } \\
\text { No } \\
\text { Yes } \\
\text { No } \\
\text { No } \\
\text { Yes } \\
\text { Yes }\end{array}$ & $\begin{array}{r}9 \\
17 \\
2 \\
3 \\
4 \\
13 \\
15 \\
6 \\
45 \\
5 \\
5 \\
4 \\
70 \\
8\end{array}$ \\
\hline Mean \pm S.D. & $47 \pm 11$ & & $75 \pm 10$ & $70 \pm 9 \cdot 3$ & $1476 \pm 327$ & & $15 \pm 19$ \\
\hline $\begin{array}{l}31 \\
32 \\
33 \\
34 \\
35 \\
36 \\
37 \\
38 \\
39 \\
40 \\
41 \\
42\end{array}$ & $\begin{array}{l}51 \\
58 \\
56 \\
44 \\
40 \\
40 \\
35 \\
48 \\
53 \\
48 \\
60 \\
46\end{array}$ & $\begin{array}{l}\text { Pyelonephritis } \\
\text { Nephrosclerosis } \\
\text { Nephrosclerosis } \\
\text { Glomerulonephritis } \\
\text { Glomerulonephritis } \\
\text { Diabetic nephropathy } \\
\text { Glomerulonephritis } \\
\text { Nephrosclerosis } \\
\text { Polycystic kidneys } \\
\text { Diabetic nephropathy } \\
\text { Glomerulonephritis } \\
\text { Glomerulonephritis }\end{array}$ & $\begin{array}{l}71 \\
73 \\
90 \\
57 \\
69 \\
50 \\
70 \\
76 \\
96 \\
86 \\
97 \\
77\end{array}$ & $\begin{array}{c}\text { Histidine Group } \\
68 \cdot 6 \\
90 \cdot 7 \\
80 \cdot 0 \\
56 \cdot 4 \\
62 \cdot 1 \\
61 \cdot 4 \\
65 \cdot 7 \\
63 \cdot 6 \\
60 \cdot 7 \\
54 \cdot 3 \\
72 \cdot 1 \\
82 \cdot 1\end{array}$ & $\begin{array}{r}1185 \\
1467 \\
1335 \\
1459 \\
1238 \\
813 \\
1441 \\
1635 \\
1096 \\
2113 \\
1273 \\
1812\end{array}$ & $\begin{array}{l}\text { Yes } \\
\text { No } \\
\text { No } \\
\text { Yes } \\
\text { Yes } \\
\text { No } \\
\text { Yes } \\
\text { Yes } \\
\text { No } \\
\text { Yes } \\
\text { Yes } \\
\text { Yes }\end{array}$ & $\begin{array}{r}6 \\
2 \\
4 \\
21 \\
7 \\
3 \\
8 \\
8 \\
6 \\
36 \\
5 \\
9 \\
72\end{array}$ \\
\hline Mean \pm S.D. & $48 \pm 8$ & & $76 \pm 14$ & $67 \cdot 8 \pm 10 \cdot 7$ & $1406 \pm 336$ & & $15 \pm 20$ \\
\hline
\end{tabular}

* Mean of all values obtained during study.
Conversion: SI to Traditional Units-Serum creatinine: $100 \mu \mathrm{mol} / 1 \approx 1.13 \mathrm{mg} / 100 \mathrm{ml}$.

dialysis patients. In dialysed patients blood specimens were obtained immediately before dialysis after a two and a half or three and a half day interdialytic interval. Patients were periodically evaluated for blood loss by careful history and chemical analysis of stools.

\section{Results}

The results are shown in table III. In both patients with chronic uraemia and those on dialysis all the baseline values shown and white cell and platelet counts were similar in both treatment groups. During the treatment period the mean haemoglobin and P.C.V. rose slightly and to a similar degree in both groups of patients on dialysis. These increases, however, were statistically significant only in the patients receiving placebo (haemoglobin, $\mathrm{P}<0.02$; P.C.V., $\mathrm{P}<0.01$ ).

No patient received blood transfusions during the study. The reticulocyte count decreased in the chronically uraemic patients on placebo, but in both the chronically uraemic patients and those on dialysis there was no difference in the reticulocyte count between the histidine and the placebo groups. The response of haemoglobin to supplementary histidine was not related to the severity of the anaemia, the plasma histidine level at the start of treatment, or the presence or absence of testosterone therapy.

Serum iron levels did not change during treatment in the chronically uraemic patients. In patients on dialysis serum iron increased only in the group on histidine, but the serum iron levels in these patients were affected primarily by losses during dialysis and the administration of iron dextran. The mean serum vitamin $B_{12}$ concentrations ( \pm S.D.) of patients on dialysis were $748+231 \mathrm{ng} / 1$ on histidine and 804 $\pm 211 \mathrm{ng} / 1$ on placebo; no patient had subnormal levels. The white cell count rose slightly only in patients on dialysis receiving placebo $(P<0.05)$ but remained within normal limits and did not differ from levels in the group receiving histidine. The platelet counts did not change during treatment in any group.

Plasma histidine decreased significantly in the chronically uraemic patients receiving placebo $(P<0.02)$ but not in those receiving histi- 
TABLE III-Effect of Administration of Placebo or L-Histidine on Haematological Values and Plasma Histidine Concentrations in Chronically Uraemic Patients and Patients on Dialysis. Results are Mean Data Collected during Baseline Period or after Six Weeks of Treatment

\begin{tabular}{|c|c|c|c|c|c|c|c|c|c|c|c|}
\hline \multirow{2}{*}{$\begin{array}{l}\text { Case } \\
\text { No. }\end{array}$} & \multirow{2}{*}{$\begin{array}{c}\text { Duration of } \\
\text { Treatment } \\
\text { Period (Weeks) }\end{array}$} & \multicolumn{2}{|c|}{ Haemoglobin $(g / d l)$} & \multicolumn{2}{|c|}{ P.C.V. $(\%)$} & \multicolumn{2}{|c|}{ Reticulocyte Count (\%) } & \multicolumn{2}{|c|}{ Serum Iron $(\mu \mathrm{mol} / \mathrm{l})$} & \multicolumn{2}{|c|}{ Plasma Histidine $(\mu \mathrm{mol} / \mathrm{l})$} \\
\hline & & Baseline & Treatment & Baseline & Treatment & Baseline & Treatment & Baseline & $\overline{\text { Treatment }}$ & Baseline & Treatment \\
\hline $\begin{array}{l}1 \\
2 \\
3 \\
4 \\
5 \\
6 \\
7 \\
8\end{array}$ & $\begin{array}{r}22 \\
26 \\
12 \\
9 \\
18 \\
12 \\
17 \\
12\end{array}$ & $\begin{array}{l}12 \cdot 1 \\
12.9 \\
10 \cdot 4 \\
10 \cdot 2 \\
10.9 \\
10.5 \\
10 \cdot 2 \\
15.9\end{array}$ & $\begin{array}{r}11.4 \\
12.6 \\
8.7 \\
10.7 \\
11.0 \\
12.9 \\
10.5 \\
14.5\end{array}$ & $\begin{array}{l}36 \cdot 6 \\
40 \cdot 2 \\
30 \cdot 9 \\
30 \cdot 1 \\
32 \cdot 5 \\
29 \cdot 8 \\
30 \cdot 8 \\
45 \cdot 9\end{array}$ & \begin{tabular}{|c|} 
Chronically $U r$ \\
$34 \cdot 2$ \\
$38 \cdot 7$ \\
$26 \cdot 2$ \\
$30 \cdot 9$ \\
$32 \cdot 3$ \\
$38 \cdot 8$ \\
$31 \cdot 8$ \\
$43 \cdot 2$
\end{tabular} & \begin{tabular}{c|} 
emic Patients \\
1.7 \\
1.3 \\
0.4 \\
0.9 \\
1.3 \\
0.8 \\
0.7 \\
0.5
\end{tabular} & $\begin{array}{c}\text { Placebo } \\
1.2 \\
0.7 \\
0.4 \\
0.6 \\
0.8 \\
0.4 \\
0.7 \\
0.5\end{array}$ & $\begin{array}{r}21.1 \\
7.7 \\
14.7 \\
11.1 \\
13.1 \\
11.5 \\
9.7 \\
13.4\end{array}$ & $\begin{array}{r}17 \cdot 4 \\
9 \cdot 1 \\
17 \cdot 0 \\
9 \cdot 7 \\
10 \cdot 7 \\
13 \cdot 4 \\
9 \cdot 0\end{array}$ & $\begin{array}{r}120 \\
70 \\
89 \\
90 \\
94 \\
81 \\
111 \\
57\end{array}$ & $\begin{array}{l}87 \\
66 \\
90 \\
82 \\
84 \\
54 \\
94 \\
53\end{array}$ \\
\hline $\begin{array}{c}\text { Mean } \\
\text { S.D. } \\
\text { P value . . }\end{array}$ & $16 \cdot 4 \pm 6 \cdot 3$ & $11 \cdot 6 \pm 2 \cdot 0$ & $s^{11 \cdot 5} \pm 1 \cdot 8$ & $34 \cdot 6 \pm 5 \cdot 8$ & $s^{34 \cdot 5} \pm 5 \cdot 4$ & $0.95 \pm 0.5$ & $0 \cdot 66 \pm 0 \cdot 2$ & $12.9 \pm 3.9$ & S. $12.4 \pm 3.6$ & $89 \pm 20$ & $.02 \pm 16$ \\
\hline $\begin{array}{r}9 \\
10 \\
11 \\
12 \\
13 \\
14 \\
15 \\
16\end{array}$ & $\begin{array}{r}22 \\
16 \\
22 \\
18 \\
12 \\
15 \\
26 \\
8\end{array}$ & $\begin{array}{r}13.1 \\
12.2 \\
7.8 \\
12.6 \\
9.3 \\
15.6 \\
10.5 \\
8.9\end{array}$ & $\begin{array}{r}12.7 \\
13.0 \\
7.0 \\
12.5 \\
8.7 \\
15.5 \\
11.0 \\
8.5\end{array}$ & $\begin{array}{l}39 \cdot 5 \\
37 \cdot 2 \\
23 \cdot 1 \\
37 \cdot 8 \\
28 \cdot 2 \\
46 \cdot 9 \\
32 \cdot 2 \\
25 \cdot 6\end{array}$ & $\begin{array}{c}\text { Chronically } \\
39 \cdot 2 \\
38 \cdot 5 \\
21 \cdot 2 \\
37 \cdot 3 \\
27 \cdot 2 \\
45 \cdot 7 \\
33 \cdot 8 \\
23.6\end{array}$ & \begin{tabular}{c|} 
raemic Patient \\
1.2 \\
0.6 \\
0.4 \\
1.4 \\
2.8 \\
0.4 \\
1.1 \\
0.9
\end{tabular} & $\begin{array}{c}\text { on Histidine } \\
0.7 \\
1.2 \\
0.7 \\
1.3 \\
2.6 \\
0.8 \\
0.6 \\
0.6\end{array}$ & $\begin{array}{r}15.8 \\
10.2 \\
10.7 \\
16.5 \\
8.8 \\
7.5 \\
10.7 \\
16.7\end{array}$ & $\begin{array}{r}16.8 \\
11.3 \\
9.5 \\
7.2\end{array}$ & $\begin{array}{r}101 \\
85 \\
78 \\
96 \\
74 \\
83 \\
79 \\
100\end{array}$ & $\begin{array}{r}80 \\
72 \\
97 \\
85 \\
80 \\
85 \\
98 \\
100\end{array}$ \\
\hline 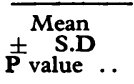 & $17 \cdot 4 \pm 6 \cdot 0$ & $11 \cdot 3 \pm 2 \cdot 6$ & $s^{11 \cdot 1} \pm 2 \cdot 8$ & $33 \cdot 8 \pm \underset{\mathbf{N}}{8 \cdot 0}$ & $s^{33 \cdot 3} \pm 8.5$ & $0.95 \pm 0.5$ & $1 \cdot 1 \pm 0 \cdot 7$ & \multicolumn{2}{|c|}{${ }^{12 \cdot 2} \pm \underset{\text { N.S. }}{11 \cdot 3} \pm 4.1$} & \multicolumn{2}{|c|}{11 N.S. $87 \pm 10$} \\
\hline $\begin{array}{l}17 \\
18 \\
19 \\
20 \\
21 \\
22 \\
23 \\
24 \\
25 \\
26 \\
27 \\
28 \\
29 \\
30\end{array}$ & $\begin{array}{l}21 \\
21 \\
18 \\
10 \\
13 \\
22 \\
12 \\
32 \\
22 \\
33 \\
18 \\
14 \\
12 \\
15\end{array}$ & $\begin{array}{r}12.0 \\
11.5 \\
10 \cdot 0 \\
5 \cdot 2 \\
9 \cdot 5 \\
9 \cdot 2 \\
9 \cdot 4 \\
7 \cdot 3 \\
8.0 \\
7 \cdot 6 \\
5 \cdot 8 \\
8 \cdot 8 \\
9.8 \\
8 \cdot 2\end{array}$ & $\begin{array}{r}11 \cdot 2 \\
12.0 \\
10 \cdot 8 \\
6 \cdot 6 \\
9 \cdot 5 \\
10.0 \\
9.7 \\
8.3 \\
8.9 \\
7 \cdot 6 \\
6.0 \\
8.9 \\
10 \cdot 6 \\
9 \cdot 2\end{array}$ & $\begin{array}{l}36 \cdot 2 \\
34 \cdot 2 \\
30 \cdot 3 \\
16 \cdot 4 \\
29 \cdot 3 \\
27 \cdot 9 \\
29 \cdot 6 \\
21 \cdot 2 \\
25 \cdot 2 \\
23 \cdot 1 \\
17 \cdot 4 \\
25 \cdot 9 \\
28 \cdot 4 \\
24 \cdot 7\end{array}$ & $\begin{array}{c}\text { Patients on } \\
33 \cdot 0 \\
35.9 \\
32 \cdot 4 \\
19.9 \\
29 \cdot 3 \\
31.5 \\
29 \cdot 3 \\
25 \cdot 2 \\
26.8 \\
22.9 \\
18.3 \\
26 \cdot 2 \\
31.5 \\
27.5\end{array}$ & $\begin{array}{c}\text { Pialysis receivi } \\
0.8 \\
1.8 \\
0.8 \\
0.4 \\
1.2 \\
2.8 \\
1.2 \\
1.7 \\
1.0 \\
0.8 \\
0.6 \\
1.5 \\
1.2 \\
1.8\end{array}$ & $\begin{array}{c}\text { Placebo } \\
1.3 \\
1.5 \\
1.0 \\
0.4 \\
1.9 \\
2.4 \\
0.8 \\
1.9 \\
0.6 \\
0.7 \\
0.5 \\
0.9 \\
1.0 \\
1.4\end{array}$ & $\begin{array}{l}16 \cdot 8 \\
15 \cdot 0 \\
17 \cdot 4 \\
20 \cdot 8 \\
10 \cdot 7 \\
27 \cdot 2 \\
7 \cdot 5 \\
16 \cdot 1 \\
20 \cdot 1 \\
18 \cdot 4 \\
22 \cdot 2 \\
16 \cdot 8 \\
26 \cdot 7 \\
17 \cdot 4\end{array}$ & $\begin{array}{l}22.9 \\
17.2 \\
15.9 \\
17.9 \\
9.0 \\
20.9 \\
17.5 \\
21.1 \\
19.7 \\
27.6 \\
25.4 \\
14.9 \\
31.5 \\
16.5\end{array}$ & $\begin{array}{r}94 \\
82 \\
49 \\
42 \\
99 \\
93 \\
71 \\
52 \\
48 \\
100 \\
105 \\
69 \\
95 \\
40\end{array}$ & $\begin{array}{r}77 \\
80 \\
65 \\
95 \\
75 \\
79 \\
60 \\
67 \\
94 \\
76 \\
50 \\
90 \\
115 \\
80\end{array}$ \\
\hline $\begin{array}{l}\text { Mean } \\
\text { S.D. } \\
\text { P value .. }\end{array}$ & $18 \cdot 8 \pm 7 \cdot 1$ & $8 \cdot 7 \pm 1.9$ & $01 \cdot 9 \cdot 2 \pm 1 \cdot 7$ & $26 \cdot 4 \pm 5 \cdot 7$ & $27 \cdot 8 \pm 5 \cdot 1$ & $1.3 \pm 0.6 \mathrm{~N}$ & $1 \cdot 2 \pm 0.6$ & \multicolumn{2}{|c|}{$19.9 \pm 5.7$ N.S. ${ }^{19.9} \pm 5 \cdot 7$} & \multicolumn{2}{|c|}{$74 \pm 24 \underset{\text { N.S. }}{ } 79 \pm 16$} \\
\hline $\begin{array}{l}31 \\
32 \\
33 \\
34 \\
35 \\
36 \\
37 \\
38 \\
39 \\
40 \\
41 \\
42\end{array}$ & $\begin{array}{l}18 \\
23 \\
18 \\
22 \\
13 \\
16 \\
10 \\
30 \\
18 \\
18 \\
13 \\
12\end{array}$ & $\begin{array}{r}10.8 \\
5.8 \\
9.4 \\
8.0 \\
5.9 \\
6.9 \\
7.7 \\
9.1 \\
14.3 \\
8.7 \\
9.9 \\
9.6\end{array}$ & $\begin{array}{r}10.2 \\
6.9 \\
9.7 \\
7.8 \\
4.9 \\
5.7 \\
9.1 \\
13.3 \\
14.4 \\
10.1 \\
10.1 \\
9.6\end{array}$ & $\begin{array}{l}34 \cdot 8 \\
17.3 \\
28.5 \\
23 \cdot 1 \\
18 \cdot 8 \\
23 \cdot 1 \\
23 \cdot 2 \\
28.8 \\
42.8 \\
26.3 \\
30.5 \\
28.9\end{array}$ & $\begin{array}{c}\text { Patients on } D \\
31.4 \\
20.2 \\
28.5 \\
23.9 \\
14.8 \\
19.1 \\
26.9 \\
39.5 \\
42.9 \\
32.2 \\
30.1 \\
28.4\end{array}$ & $\begin{array}{c}\text { lysis receiving } \\
2.4 \\
1.0 \\
0.9 \\
1.4 \\
1.5 \\
3.1 \\
1.5 \\
1.2 \\
0.9 \\
0.9 \\
1.6 \\
1.4\end{array}$ & $\begin{array}{c}\text { Histidine } \\
1.5 \\
1.1 \\
0.8 \\
1.1 \\
0.7 \\
1.2 \\
1.2 \\
0.4 \\
1.3 \\
2.0 \\
1.9\end{array}$ & $\begin{array}{r}17.4 \\
25 \cdot 1 \\
16 \cdot 1 \\
22 \cdot 6 \\
35.3 \\
9.1 \\
24 \cdot 2 \\
13.4 \\
13.4 \\
14 \cdot 1 \\
16.3 \\
10.9\end{array}$ & $\begin{array}{l}13.6 \\
38 \cdot 1 \\
18 \cdot 6 \\
25.4 \\
31.3 \\
13 \cdot 2 \\
35.4 \\
26 \cdot 1 \\
11.3 \\
21.8 \\
16.1 \\
22.9\end{array}$ & $\begin{array}{r}42 \\
58 \\
52 \\
65 \\
61 \\
55 \\
87 \\
68 \\
64 \\
105 \\
74 \\
53\end{array}$ & $\begin{array}{r}37 \\
96 \\
82 \\
77 \\
81 \\
44 \\
156 \\
83 \\
62 \\
130 \\
77 \\
120\end{array}$ \\
\hline $\begin{array}{c}\text { Mean } \\
\text { S.D. } \\
\text { P value .. }\end{array}$ & $17 \cdot 6 \pm 5 \cdot 5$ & $8 \cdot 8 \pm 2 \cdot 3 \mathrm{~N}$ & $9 \cdot 3 \pm 2 \cdot 8$ & $27 \cdot 2 \pm 7 \cdot 0_{\mathrm{N}}$ & S. $28 \cdot 2 \pm 8 \cdot 1$ & $1.5 \pm 0.7$ & s. $1.2 \pm 0.5$ & $18 \cdot 1 \pm 7 \cdot 3$ & 05 & $64 \pm 17$ & $05^{87} \pm 34$ \\
\hline
\end{tabular}

Conversion: SI to Traditional Units-Serum Iron: $1 \mu \mathrm{mol} / 1 \approx 5.6 \mu \mathrm{g} / 100 \mathrm{ml}$.

Histidine $v$. placebo for either chronically uraemic patients or patients on dialysis, $P=$ not significant for each column.

dine. In the patients on dialysis plasma histidine rose only in those receiving histidine $(P<0.001)$.

During the treatment period the mean daily protein intakes ( \pm S.D.) of the chronically uraemic patients on histidine and placebo were about $64+24 \mathrm{~g}$ and $66 \pm 19 \mathrm{~g}$ respectively. In the patients on dialysis the daily protein intake was about $75 \pm 20 \mathrm{~g}$ and $75 \pm 16 \mathrm{~g}$ for the histidine and placebo groups respectively. The chronically uraemic patients were estimated to have ingested a mean of $1500 \mathrm{mg} /$ day of histidine in their food and those on dialysis $1700 \mathrm{mg} /$ day.

At the end of treatment four patients on dialysis whose haemoglobin levels had remained low (cases 24, 26, 32, and 35) were given Lhistidine $4 \mathrm{~g}$ /day for a mean of 14 additional weeks (range 10-25 weeks). When the code was broken we learnt that two of these patients had already been receiving histidine. The haemoglobin and P.C.V. did not change in any patient, though in the two who had previously received placebo (cases 24 and 26 ) the plasma histidine increased from a mean $71 \mu \mathrm{mol} / 1$ to $103 \mu \mathrm{mol} / 1$.

\section{Discussion}

Our results indicate that dietary supplementation with Lhistidine does not increase the haemoglobin or P.C.V. in chronically uraemic patients or those on dialysis. In the latter these values increased significantly only in those receiving placebo, while patients taking histidine experienced a rise of similar magnitude which was not significant. Moreover, improvement in anaemia was very slight in both groups. Jontofsohn et al. ${ }^{10}$ recently presented evidence that histidine does not improve the anaemia of uraemia.

The increase in haemoglobin in the dialysis patients may have been related to the fact that data collected during superimposed illness or substantial blood loss were excluded from analysis, but the alternative to excluding such data would have been to increase greatly the variance of such data. Consequently, the response to supplementary histidine would have to have been evaluated in many more patients. In patients on dialysis the quantity of blood lost from sampling (about $25 \mathrm{ml} /$ week), retention in the dialyser $(6 \mathrm{ml})$, and clotted or leaking dialysers (combined incidence about $6 \%$ ) was similar in both groups. Hence the degree of blood loss in the placebo and histidine groups probably could not have affected our results.

The rise in plasma histidine in the patients on dialysis receiving supplementary histidine confirms our observations on the direct correlation between the plasma level and dietary intake of this amino-acid. ${ }^{11}$ The failure to observe a rise in 
plasma histidine in the chronically uraemic patients who were treated with histidine might have reflected decreased histidine intake from food taken during treatment. Renal function decreased during the treatment period in these patients, and their protein intake was reduced. The finding that plasma histidine levels decreased in the chronically uraemic patients receiving placebo supports this possibility.

Histidine is an amino-acid of particular importance for erythropoiesis. There is abundant evidence that diets deficient in histidine are associated with decreased erythropoiesis, though how histidine promotes erythropoiesis is unknown. ${ }^{2}{ }^{312}$ It may be pertinent that histidine is an amino-acid which is particularly abundant in the haemoglobin molecule and provides the linkage for iron in the haem moiety to globin. It is not clear why the anaemia did not improve in the patients receiving histidine. Their plasma histidine levels during the baseline period were at least as great as those of normal people ingesting 40-60 g protein diets, ${ }^{13}$ and they may have had adequate body histidine pools. Hence the administration of histidine in quantities sufficient to prevent a deficiency possibly promotes erythropoiesis, but additional histidine does not further enhance red-cell production. The relation between histidine and erythropoiesis may thus be similar to that between iron or folic acid and red-cell production.

The amount of dietary histidine necessary for a maximum effect on erythropoiesis is unknown. Our patients took about $1500-1700 \mathrm{mg} /$ day of histidine in their food. The quantity necessary for maximal erythropoiesis is probably not greater than this since the patients who received supplementary histidine showed no improvement in their anaemia. Our results do not exclude the possibility that supplementary histidine may promote erythropoiesis in patients with chronic uraemia or on dialysis who are poorly nourished and eating less than the
64-75 g/day of protein taken by our patients. Nevertheless, our results do suggest that the anaemia in such patients may improve as much by increasing the nutritional value of the diets as by giving supplementary histidine.

We thank Dr. Jack Coburn for reviewing the manuscript, Mrs. Betty Card, Mrs. Julia Tallos, Mrs. Donna Weaver, Mrs. Carolyn Schaefer, Mr. Jerry Gault, and the Veterans Administration Pharmacy Service for their help.

This study was supported by the Artificial Kidney-Chronic Uremia Program, D.H.E.W. Contract No. 1-AM-3-2210 and the Kidney Foundation of Southern California. Veterans Administration Project No. 5016-01.

\section{References}

1 Bergström, J., et al., Life Sciences (Part II), 1970, 9, 787.

Giordano, C., et al., in Uremia: An International Conference on Pathogenesis, Diagnosis and Therapy, ed. R. Kluthe, G. Berlyne, and B. Burton, p. 138. Stuttgart, Georg Thieme Verlag, 1972.

3 Kopple, J. D., and Swendseid, M. E., Journal of Clinical Investigation, 1975, 55, 881 .

4 Giordano, C., et al., in Proceedings of 3rd International Congress of Nephrology, Washington 1966, ed. G. E. Schreiner, Vol. 3, p. 214. Basel, Karger, 1967

5 Giordano, C., et al., British Medical fournal, 1973, 4, 714.

${ }_{6}$ Giordano, C., et al., Dialysis, Transplantation, Nephrology: Proceedings of the Tenth Congress of the European Dialysis and Transplant Association, of the Tenth Congress of the European Dialysis and Transplant

'Blumenkrantz, M. J., et al., Proceedings of Clinical Dialysis and Transplant Forum, 1973, 3, 117.

8 United Nations Food and Agriculture Organization, Amino Acid Content United Nations Food and Agriculture Organization, Amino Acid Cont
of Foods and Biological Data on Proteins. Geneva, F.A.O., 1972.

9 U.S. Department of Agriculture, Handbook 8, Composition of Foods. U.S. Department of Agriculture, Handbook 8, Composition
Washington, U.S. Government Printing Office, 1963.

10 Jontofsohn, R., et al., Kidney International, 1974, 6, 6A.

Kopple, J. D., Paniagua, M., and Swendseid, M. E., Proceedings of the American Society of Experimental Biology, 1975, 34, 931.

12 Sebrell, W. H., and McDaniel, E. G., fournal of Nutrition, 1952, 47, 477.

${ }^{13}$ Swendseid, M. E., and Kopple, J. D., unpublished observations.

\title{
Emergence of Group B Streptococci in Obstetric and Perinatal Infections
}

\author{
T. M. S. REID
}

19/1000; maternal and neonatal morbidity rates of 16 and $2 \cdot 7 / 1000$, respectively; and an overall neonatal mortality of $1 / 1000$ live births.

\section{Summary}

$A$ retrospective study of obstetric and perinatal illness due to group $B$ streptococci during 1972-4 based on bacteriological referrals from Aberdeen Maternity Hospital and Special Nursery disclosed (1) a wide spectrum of maternal morbidity, particularly associated with amniotomy and a prolonged rupture-delivery interval, and (2) the emergence of the group B streptococcus as a major cause of serious neonatal infection in infants of low birth weight, often in the absence of maternal pyrexia. The group $B$ isolates appeared to show a previously undocumented increased resistance to the aminoglycosides gentamicin and kanamycin.

A prospective study of 369 random deliveries in Aberdeen Maternity Hospital showed a group B vaginal carriage rate of $49 / 1000$; a neonatal colonization rate of

University Department of Bacteriology, Aberdeen AB9 2ZD

T. M. S. REID, M.B., B.MED.BIOL., Lecturer

\section{Introduction}

The group B streptococcus (Streptococcus agalactiae) is classically associated with bovine mastitis. Human vaginal carriage of group B strains was documented by Lancefield and Hare, ${ }^{1}$ who found a $2.3 \%$ carriage rate in the puerperium with a $20 \%$ incidence of minor maternal infective complications. With the introduction of penicillin for streptococcal infections few reports of group B streptococci appeared until Hood et al. ${ }^{2}$ and Eickhoff et al. ${ }^{3}$ in the U.S.A. showed them to be preeminent in perinatal infections. Many reports have shown the insidious nature of "early-onset" septicaemic and "late-onset" meningitic forms of the infection in neonates in the U.S.A. ${ }^{4-8}$ and Europe.? meningitis in Britain, but though official notifications have warranted epidemiological comment ${ }^{2} 10$ the group B streptoIn 1974, when we were confronted with an apparently explosive retrospectively the obstetric and perinatal morbidity based on
Jones and Howells ${ }^{8}$ reported the first two cases of group B coccus appears to be a much underrated pathogen for man. increase in group-B-related diseases, we attempted to determine 